\title{
A Smart Reader for Blind People
}

\author{
L Latha, V Geethani, M Divyadharshini, P Thangam
}

\begin{abstract}
This paper presents an intelligent bot for aiding the visually challenged people. Presently, $81 \%$ are visually impaired who live in the developing countries. Nowadays Human communication is mainly focused on text and speech. To read the text a human needs a vision. Survey conducted on several papers and systems provides hardware consisting of a camera interface with Raspberry Pi for processing the text. The camera captures text image of a handwritten or printed text. The raspberry pi makes use of Optical Character Recognition (OCR) software installed in it, to perform the conversion of an image to text and similarly text to speech conversion. The assistant is applicable for visually impaired people as well as for normal people in order to increase their level of comfort.
\end{abstract}

Keywords: Text to speech, Image to Text, Optical Character Recognition, Raspberry Pi, Speech output, Python Programming.

\section{INTRODUCTION}

The World Health Organization (WHO) survey says that over 285 million people are estimated to be visually challenged worldwide and blind individuals around fifty million worldwide. Although there are many solutions to help individuals who are blind to read the content, but still it requires a lot of enhancement for better reading ${ }^{[1]}$. The first reference paper named Book reader is an assistive reading system based on the usage of camera for visually impaired people for reading the text on the hard-copied documents, printed text labels and products. This work extracts text from image and then converts it to voice. This can be developed in a Raspberry pi with a battery backup, and this smart reader will be very useful for visually impaired people and also useful for normal human beings.

The existing technology poses a lot of challenges to the visually impaired people in accessing the written text or hard-copied text. Also problems like mobility, alignment issues exist. So a device is required that assists the needy people to read the text effectively.

\section{BLOCK DIAGRAM}

The block diagram of the book reader ${ }^{[1]}$ system is shown in Figure 1. The written text is placed underneath a camera by the individual to check for fine quality of the image. An assistive system provides text localization algorithm with some precision. On startup, the system checks if all devices and their connectivity is correct. The graphical user interface shows the status of the clicked image. The Raspberry Pi is an embedded system which has integrated peripheral

Revised Manuscript Received on 14 August, 2019.

L Latha, Professor, Computer Science and Engineering, Kumaraguru College of Technology, Coimbatore, Tamilnadu, India.

V Geethani, Student, Computer Science and Engineering, Kumaraguru College of Technology, Coimbatore, Tamilnadu, India.

M Divyadharshini, Student, Computer Science and Engineering, Kumaraguru College of Technology, Coimbatore, Tamilnadu, India

P Thangam, Asso. professor, Electrical \& Computer Engineering, King Abdulaziz University, SaudiArabia. devices like Bluetooth, Wi-fi USB, analog-digital conversion etc.,

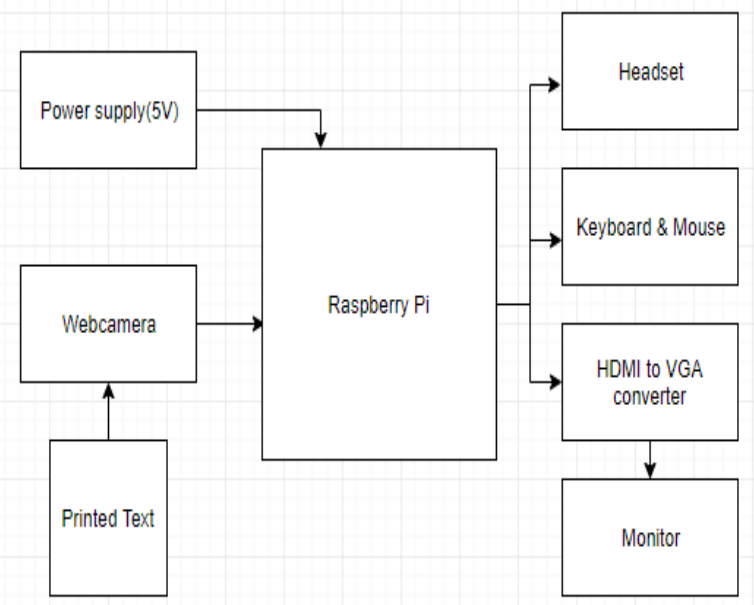

Fig 1: Block diagram representation ${ }^{[1]}$

\section{OBJECTIVE}

Though various types of reading assistants are present for the visually challenged persons, based on the references, there doesn't exist special reading assistant which can read the background and challenging patterns available among the text present in the printed materials or books ${ }^{[2]}$. Those patterns can be of many kinds with different fonts, colours, scales and multiple orientations.

The objective mainly focuses in fulfilling the need of visually impaired people in reading and browsing text from hand-held objects like books, documents etc., by implementing a camera-based text reading framework which tracks the area containing the text and extracts them from the article or book within the camera view. The planned algorithmic program is effectively accustomed to handle completely different background patterns, and extract text data from any reasonably hand-held objects or books. Nowadays people getting more tired of reading large number of pages and also a person is needed to help visually challenged people to read text loud and to explain them while we build this anyone can hear the text.

\section{METHODOLOGY}

The design usually involves a fully integrated system which feeds the printed text as input through camera for digitization and the OCR (Optical Character Recognition Engine), a software module processes the scanned document $^{[3]}$. 
A methodology for recognizing the sequence of characters present and also to indicate the current reading line is enabled ${ }^{[1]}$. OpenCV (Open source Computer Vision) libraries are used for capturing the image of text and to perform the character recognition etc.

The architecture of the smart reader is shown in the Fig: 1 where the plastic sheet indicates the text to be read. Optical character recognition (OCR) is the technology used for translating a captured image of written text into machineencoded text. ${ }^{[3]}$

In order to reduce storage space digitizing texts is helpful ${ }^{[5]}$. As the editing and publication of text documents written on a paper are time taking, it is widely used in storage and document analysis, especially to convert the documents into electronic files. The OCR technology then makes use of methods like machine translation, text-tospeech, etc to capture a page. Finally the recognized text document is fed to the output device.

\section{FLOW OF PROCESS}

Figure 2 illustrates the flow of process of the method. The process flow describes the steps concerned in recognizing the text from the given image and process the text to get the specified output within the form of voice as shown in Fig: 2.

1.The built-in camera captures the text image by moving the device over the printed page that is thus known as image capturing ${ }^{[4]}$.

2. There are three steps in Pre-processing ${ }^{[5]}$ : they are a.Skew correction, b.Linearization and c.Noise removal. The captured image is initially checked for skewing. There are two possibilities of orientation i.e., left or right orientation for image skewing, during which the image intensity is increased and then it is binarized.

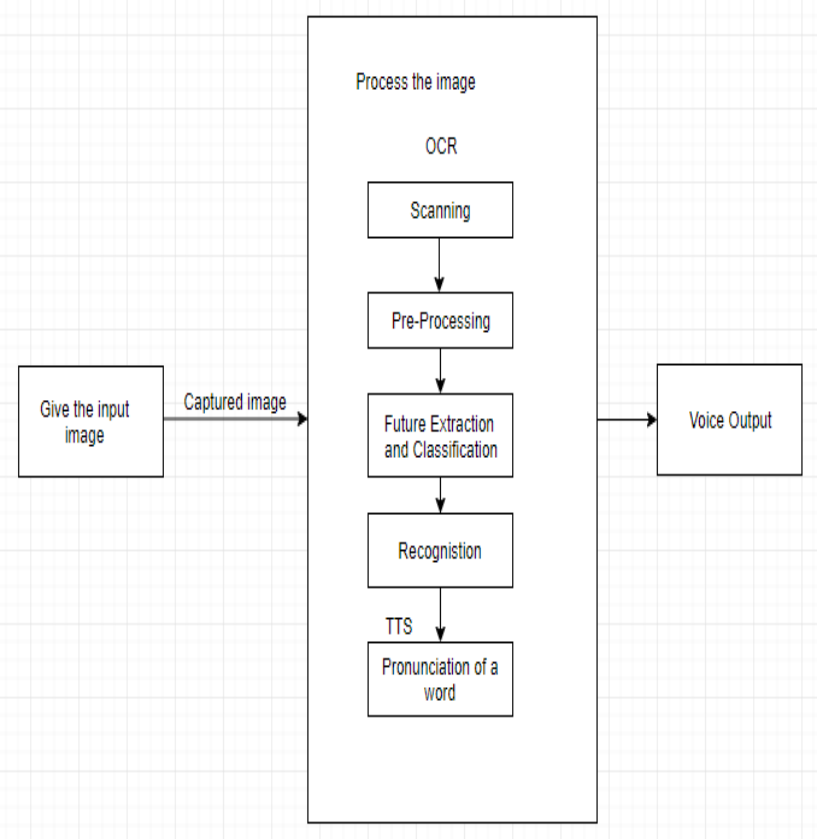

Fig 2: Process Flow

${ }^{[2]}$ The process of skew detection is implemented to perform certain functions like angle of orientation which is to be between \pm 15 degrees, and if the result is true then a simple image rotation is applied, that successively produces a skew corrected image.
3. In Segmentation, the inter line spaces is checked for the binarizedimage ${ }^{[6]}$. On detection of inter line spaces present in the image, across the interline gap it is divided into sets of paragraphs. With regard to the background, to sight the width of horizontal lines, histogram of the image is employed. For vertical space intersection the lines are scanned vertically.

4. Feature extraction, here the first character glyph is defined with a set of attributes such as Height and width of the character, Numbers of horizontal and vertical lines present, Numbers of circles present in the various regions ${ }^{[8]}$.

5. The Raspberry Pi board processes the ASCII values of the characters for Image to Text conversion ${ }^{[4]}$. The normalized text transcription is saved by matching each of the character with its corresponding template.

6.A minimally filteredPWM output generates the image to speech conversion output ${ }^{[2]}$. To improve the sound quality and volume a USB audio card can also be used. There are two options of attaching a microphone into Raspberry Pi. One is through USB mic, another to have an external USB sound card.

\section{HARDWARE IMPLEMENTATION\& RESULTS}

The system setup includes specific set of hardware components which include:

Raspberry Pi which can be thought of as a small computer that plugs into the monitor and connects to a keyboard. The basic hardware components in Raspberry $\mathrm{Pi}$ are the power source, memory and I/O. The common features included in different versions of raspberry pi are $1.2 \mathrm{GHz}$ 64-bit quadcore ARMv8 CPU, Camera interface (CSI) and Display Interface (DSI).

One example for hardware setup is shown in fig-3. A cameracaptures the images in real time and feeds it to a computer. A power supply device supplies or voltage to the output loads. A-regulated output voltage of $+5 \mathrm{v}$ with output current of $100 \mathrm{~mA}$ is generated.

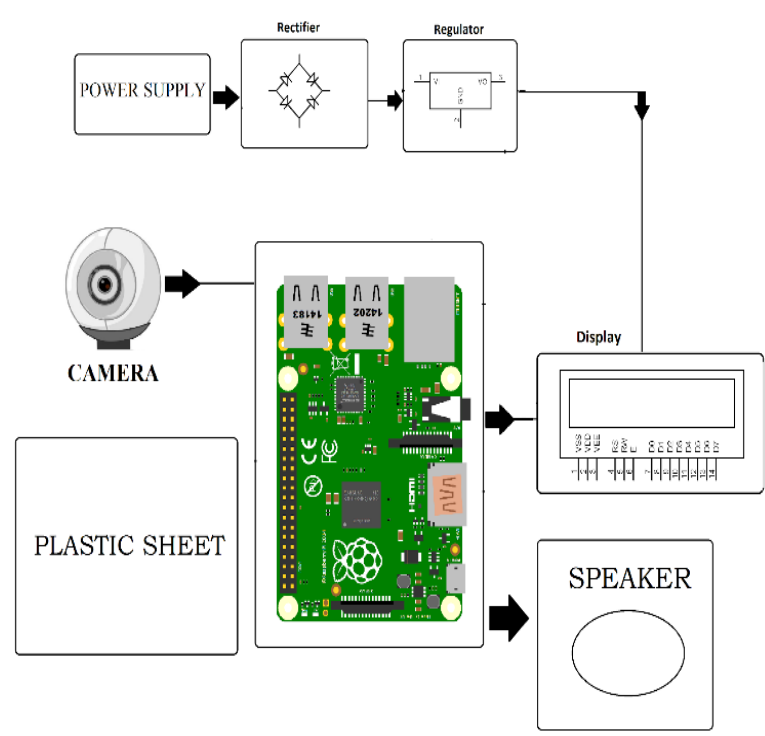

Fig- 3: Architecture diagram

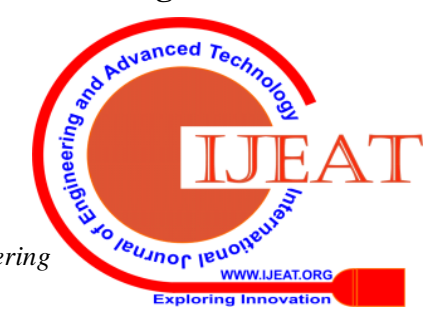




\section{CODE IMPLEMENTATION}

Similarly, the code implementation shares some common feature such as operating system, language, image extracting process and the library for further processing.

Operating system:Raspbian (Debian)

Language: Python 3.6

Platform: Tesseract, OpenCV (Linux-library)

Library: OCR engine, TTS engine

Python language is a script language is used for writing the algorithm. OpenCV library is used for calling the functions in the algorithm.

\section{CONCLUSION}

According to the survey made through the reference papers, the smart reader for blind produces a positive outcome when applied in practical world. Since the OpenCV platform is used for text detection, it is very handy and convenient to use compared to the PC platform. This system is useful for visually impaired persons to access information which is in the form of documents, texts or printed forms. The future implementation may include accuracy and fulfil the limitations of existing systems.

\section{REFERENCES}

1. AmalJojie, Ashbin George, DhanyaDhanalalNayana J, Book Reader for Blind, IOSR Journal of Engineering (IOSRJEN)

2. S. Aditi, SP. Annapoorani, A. Kanchana, Book Reader Using Raspberry Pi for Visually Impaired, International Research Journal of Engineering and Technology (IRJET), Volume 05, Issue 03, March 2018

3. KA.Aslam,TanmoyKumarRoy, Sridhar rajan, T. Vijayan, B. KalaiSelviAbhinayathri, Smart Reading System for Visually Impaired People, International Journal of MC Square Scientific Research, Volume 09, Issue 02, 2017

4. V.Ajantha Devi, Dr. S SanthoshBaboo, Embedded optical character recognition on Tamil text image using Raspberry $\mathrm{Pi}$, International Journal of Computer Science Trends and Technology (IJCST), Volume 02, Issue 04, Jul-Aug 2014

5. MallapaD. Gurav, Shruti S. Salimath, Shruti B. Hatti, Vijayalaxmi I. Byakod, B-LIGHT: A Reading aid for the Blind People using OCR and OpenCV, International Journal of Scientific Research Engineering \& Technology (IJSRET), Volume06, Issue 05, May 2017

6. S.Rajakumar, SubbiahBharathi, Century Identification and Recognition of Ancient TamilCharacter Recognition, International Journal of Computer Applications, Volume 26, Issue04, July 2011.

7. Rahul R. Patil, Audumbar R. Misal, Ketan R. Nalawade, Survey paper on Text Recognition Using Image Processing, International Journal of Advanced Research in Electronics and Communication Engineering (IJARECE), Volume 04, Issue 03, March 2015.

8. Praveen Choudhary, Dr.Vipin Kumar Jain, Text Extraction from an Image by using Digital Image Processing, International Research Journal of Computer Science (IRJCS), Volume 05, Issue 07, July 2018.

9. Nagaraja L, Nagarjun R S, Nishanth M Anand,Nithin D, Veena S Murthy, Vision based Text Recognition using Raspberry Pi, International Journal of Computer Applications, National Conference on Power Systems \& Industrial Automation (NCPSIA), 2015.

10. AnushGoel, AkashSehrawat, AnkushPatil, PrashantChougule, SupriyaKhatavkar,Raspberry $\mathrm{Pi}$ Based Reader for Blind People, International Research Journal of Engineering and Technology (IRJET), Volume05, Issue 06,June2018
11. D.Velmurugan,M.S.Sonam,S.Umamaheswari ,S.Parthasarathy, K.R. Arun, A Smart Reader for Visually Impaired People Using Raspberry PI, International Journal of Engineering Science and Computing (IJESC), Volume 06, Issue 03, 2016

12. Agalya, A., B. Nagaraj, and K. Rajasekaran. "Concentration control of continuous stirred tank reactor using particle swarm optimization algorithm." Trans EngSci 1, no. 4 (2013): 57-63.

13. Aaron James S, Sanjana S, Monisha M, OCR based automatic book reader for the visually impaired using Raspberry PI, International Journal of Innovative Research in Computer and Communication Engineering, Volume 04, Issue 7, January 2016.

14. AbhijithShaji, AbhishekAravindan, NishamRafeeque, Naveen K K, Reading assistant for visually impaired people, International Research Journal of Engineering and Technology (IRJET), Volume 05, Issue 04, April 2018.

15. Esra Ali Hassan, Esra Ali Hassan, Smart Glasses for the Visually Impaired People, Computers Helping People with Special Needs, 15th International Conference, ICCHP, July 2016. 